\title{
Language Ideologies and Saudi Society: Understanding the Notion of Diglossia
}

\author{
Abdullah Abdulrahman Bin Towairesh ${ }^{1}$ \\ ${ }^{1}$ Department of English Language and Translation, King Saud University, Riyadh, Saudi Arabia \\ Correspondence: Abdullah Abdulrahman Bin Towairesh, Department of English Language and Translation, King \\ Saud University, Riyadh, Saudi Arabia. E-mail: abintowairesh@ksu.edu.sa
}

Received: April 6, 2020

Accepted: May 8, $2020 \quad$ Online Published: May 14, 2020

doi:10.5539/ijel.v10n4p90

URL: https://doi.org/10.5539/ijel.v10n4p90

\begin{abstract}
Discussing the role of Spoken varieties within Saudi society exposes a point of tension between those who view these varieties as a threat to Fus 'ћa Arabic and those who value them for their close association with local traditions and culture. The absence of a clear understanding of the concept of diglossia among the general public is at the core of this issue. Thus, one can see that although the use of Spoken varieties is expanding rapidly through new mediums such as "Shilat" (folkloric singing) and "Alqanawat Ash-ShaSbeyah" (TV channels focusing on folklore), the linguistic campaigns that criticize these varieties remain as strong as ever. In this context, this paper aims to explore the discrepancy between linguistic ideologies within society and the reality of language use on the ground. The data used in this study were collected from questionnaires disseminated among Saudi speakers from both sexes and different age groups. The qualitative and quantitative analyses of the data reveal a number of directions and views that are prevalent within Saudi society regarding the H/L dichotomy. There seems to be a wide consensus about accepting Spoken varieties as a normal component of the linguistic repertoire of speakers, provided that such varieties are used in their predetermined domains. In contrast, any signs of infringement on the functions reserved for Fus' ha Arabic are always condemned and denounced. These infringements include the nonstandard use of language in any written form, such as the use of local varieties on internet forums, newspapers, and magazines or on information websites, like Wikipedia. This paper also examines the participants' views on using Spoken Arabic on social media platforms, and their attitudes towards the influx of recent English borrowings into Spoken Arabic.
\end{abstract}

Keywords: language ideologies, spoken Arabic, diglossia, linguistic awareness

\section{Introduction}

Fus `ha Arabic holds an undeniable presence in the conscience and lives of Arabs all over the world. It has an indisputable position in the Arab world, and it is the language of the most sacred scripts in the Islamic religion, which are the Holy Quran and the Prophet's Sunnah. Fus 'ha Arabic symbolizes the ages of innovation and power in the history of its speakers and represents, in their view, the right path to regaining cultural and scientific revitalization. Spoken Arabic, in contrast, is sometimes perceived as a threat to Fus 'ha Arabic, and this view has strong support within large sections of the population in Arab societies. The local attitudes towards Spoken Arabic, however, differ from one society to another. Many Egyptians, for example, describe their Spoken Arabic as beautiful, and view it as "easy, light, full of humor, and more beautiful than other Arabic dialects" (Haeri, 2003, p. 37). It is also referred to as a "true symbol of local national culture" (Haeri, 2000, p. 63). These opinions, however, are not echoed within Saudi society, at least not loudly, as will be explained in detail later in this paper. Nonetheless, the use of Spoken varieties is expanding in real life, to the point of moving into domains that once used to be reserved exclusively for Fus' $\hbar$ a Arabic. This paper investigates the discrepancy between language ideologies and the realities of language use in real life.

The contentious discussions about the use of language in Saudi society usually merge into one dominant view that revolves around the imminent threat to Fus ${ }^{\circ}$ a (Classical/Standard) Arabic from Spoken varieties. Local magazines and newspapers are full of articles, essays, and opinion pieces that lament the current state of Fus ${ }^{\circ}$ a and blame Spoken varieties for this circumstance. These writings predict dire consequences if society does not change the way it deals with language. The proponents of these views, however, usually ignore the diglossic nature of Arabic societies and the fact that diglossia is a stable phenomenon that can be found in several other 
speech societies, as described by Ferguson (1959). The absence of a clear understanding of the linguistic situation among the wider public results in a constant feeling of guilt, because people believe they are corrupting their language when using Spoken Arabic.

Media stories that blame Spoken varieties for the decline of Fus' ha have appeared in major Saudi newspapers and magazines. These include Riyadh Newspaper (“Arabic Language, Problems and Challenges", Sept. 4, 2013), Alwatan Newspaper ("Islamic University Conference Addresses Linguistic Challenges", April 2, 2015), and Almarefah Magazine ("Is the Media Affecting Arabic Language Negatively", Oct. 30, 2014). In these news articles and opinion pieces, Spoken Arabic is listed as one of the main challenges to Fus' $\pitchfork a$ and as an obstacle to the revitalization of the Standard.

In contrast, there are two recent developments that are closely connected to the use of Spoken Arabic and that have given an unprecedented boost to the popularity and influence of Spoken varieties in Saudi society. The first of these two developments is the proliferation of recording and listening to the art of "Shilat," a type of local singing that sounds like regular music but does not require the use of musical instruments. This is achieved by using human voices to produce tunes and rhythms. The recent increase in the popularity of Shilat has been attributed to several factors: (a) believing it is a permissible alternative to music, which some understand to be prohibited (based on certain interpretations of religious texts), (b) the simplicity in its production, (c) the demand for it during cultural and national festivals, and (d) the absence of music concerts during a certain period in the society's history (Al-Shahrani, 2016). Shilat songs are almost exclusively delivered in Spoken Arabic, which has presented a new and powerful platform for local varieties.

The second development is the spread of local TV channels that specialize in traditional poetry, folkloric dances, and in covering traditional events like tribal gatherings, camel festivals, and camel races. These channels (referred to locally as "Alqanawat Ash-ShaSbeyah" or folklore TV channels) have become a phenomenon in society and have experienced strong viewership numbers. As a testament to the effects these channels are having on Saudi society, the government's official dialogue center (King Abdul-Aziz Centre for National Dialogue, or KACND) held a discussion panel that focused on exploring the negative and positive aspects of the spread of these channels.

The result of the dilemma represented in this dichotomy is the emergence of a society that seems to deplore Spoken Arabic on the record, in certain local academic conferences, and on media outlets, but at the same time cannot satisfy the insatiable appetite of the wider public for entertainment delivered in those varieties. However, in general, speakers are able to distinguish between the different roles associated with the $(\mathrm{H})$ and $(\mathrm{L})$ varieties in the diglossic Saudi society. Thus, one would see an overall acceptance of the use of Spoken varieties for purposes that have historically been in the (L) domain, such as traditional poetry and singing (as in Shilat), and conversations about camel breeding and racing (like the ones featured on the Alqanawat Ash-ShaSbeyah or folklore channels). In contrast, members of society would usually reject the infringement of Spoken varieties on areas that are usually reserved for the $(\mathrm{H})$ variety. This includes opposing the use of Spoken Arabic in the press (both in print and online) and in writings about scientific issues, as will be explained in detail later in this paper. Nonetheless, the continuous campaign against Spoken Arabic might be the reason behind the mixed feelings some speakers have about the language they use. The constant demonization of Spoken varieties might lead some members of the public to believe that it is their duty to stand against any use of such varieties and to call for a Fus `ha-only society, even though they themselves might struggle when using Fus `ha.

\section{Aims of This Paper}

This paper aims to present a clearer picture of the linguistic situation in Saudi society, especially in the area of language ideologies and attitudes. In addition, it is hoped that this work will help in exploring the expressed attitudes towards Spoken Arabic, and in matching these attitudes with the realities of the current linguistic situation on the ground. These realities include the expanded use of Spoken Arabic in media outlets and on social networking platforms. In other words, the goal is to find answers to this question: Why do we still see signs of the strengthening status and role of Spoken Arabic, despite years and years of campaigning against such varieties, and despite critics' holding them responsible for the decline of Fus 'ha?

Although the data on the age, gender, and regional background of the participants was originally collected in the data collection stage, the age factor has proven to be the most significant indicator for determining the differences between participants in responding to the questionnaire. Thus, this factor will be the focus of discussions in this paper and will help shed more light on the roots of linguistic attitudes in this region, as well as their direction of growth. Finally, this paper contributes to the discussion on the threats facing Fus'tha and provides an argument that does not gloss over the reality of diglossia and that presents the possibility of 
coexistence between Fus `ha and Spoken varieties, without the need for one to eliminate the other.

For the purposes of this paper, the term "Fus" ћa" Arabic will be used to refer to all the varieties that can represent the $(\mathrm{H})$ position within the definition of diglossia. These include Modern Standard Arabic (MSA) and Classical Arabic. Currently, MSA is the actual $(\mathrm{H})$, but the aim was to use a term that is inclusive of all the possible manifestations of $(\mathrm{H})$ and recognizable to research participants.

\section{Background and Related Literature}

The current linguistic scene in Saudi society can be described as being part of the greater diglossic situation found in Arabic-speaking societies. As explained by Ferguson (1959), the (H) variety in these societies is Fus 'ha (the language of the Quran, the holy book of the Islamic faith), whereas the (L) represents the different spoken dialects used all over the Arab world. There are, however, general notions about how these varieties are viewed within society, including the idea that the (L) varieties are simplified and corrupt forms of the Standard, and that the $(\mathrm{H})$ is always more complex and eloquent (Suleiman, 2004; Versteegh, 2001).

It is, however, difficult to find literature that directly addresses the issue of attitudes towards the existence of (L) varieties or language ideologies within Saudi society. This includes the lack of information on the discrepancy between expressed attitudes and linguistic practices on the ground. Most of the studies that address Spoken Arabic in Saudi Arabia approach this issue from a dialectological perspective, or with the aim of studying the attitudes of speakers of one variety towards other varieties of Arabic. These studies, however, do not discuss public opinions about the existence of an (L) variety as a whole or the levels of agreement with the calls to limit the influence of $(\mathrm{L})$ within society.

Nonetheless, these studies are important, because they show the possibilities and vibrancy of local Spoken varieties and the important functionalities associated with them. One of these studies discusses the attitudes of Urban Meccan Hijazi Arabic (UMHA) speakers towards their dialect. In this paper, Alahmadi (2016) used a 5-point Likert scale questionnaire to determine the influence of social factors (e.g., age, sex, and educational level) on the way speakers perceive their own dialect. The results of this study demonstrated that UMHA speakers generally have positive attitudes towards their dialect, regardless of age, sex, or educational status.

Other studies on the issue of Spoken varieties within Saudi society include Alrumaih's (2002) work on "Najdi perception of Saudi regional speech." In this study, the researcher investigated the attitudes of Najdi Arabic speakers towards other dialects in Saudi Arabia, comparing them with their attitudes toward their own dialect. In addition, Alrumaih asked his participants to draw dialectical boundaries on a map to show their awareness of the different speech varieties within Saudi society. Aldosaree (2016) also examined speakers' attitudes toward Saudi dialects, using informants who come from different linguistic backgrounds (i.e., not from one specific Saudi region).

In addition to the newspaper reports and articles that have laid the blame on Spoken Arabic for the decline of the Standard, the authors of some academic papers have presented similar propositions. This includes, for example, AlMuazen (2016), Khater-Aso (2012), and Wadgiri (2012). In these papers, the authors tried to reinforce the arguments that Spoken Arabic is the main reason behind the decline of Fus' ha, and that the speakers are responsible for this decline. This responsibility is the basis for the constant feeling of guilt that Arabic language speakers have towards their language.

The view that Fus ' ha is endangered and that spoken varieties are the source of the danger partially constitute the linguistic ideologies in Saudi society. However, what do we actually mean when we talk about language ideologies? Linguistic or language ideologies can be described as "sets of beliefs about language, articulated by users as a rationalization or justification of perceived language structure and use," and these beliefs usually come loaded with moral and political considerations (Woolard \& Schieffelin, 1994, p. 57). Therefore, these ideologies will determine "which linguistic features get selected for cultural attention and for social marking" (Schieffelin \& Doucet, 1998, p. 285). In the case of Arabic-speaking societies, the moral considerations draw upon the strong association between Fus' ha Arabic and Islam, and the political considerations are based on the association between Arabic language and certain historical events, such as independence from colonialism, and the calls for Arab unity and nationalism. Although Saudi society is part of the Arab World, the moral considerations behind linguistic ideologies manifest themselves more clearly than do the political considerations.

\section{Methodology}

This paper discusses the various linguistic ideologies prevalent in Saudi society, especially with regard to the juxtaposition between the way people use the Standard and the Spoken varieties and the way they view them. To achieve the goals of this study, an electronic questionnaire was designed to collect data on language use and 
ideological stances. The questionnaire included both open-ended and multiple-choice questions, covering different aspects of the topic, including support for research on Spoken varieties, views on publications and advertisements that employ such varieties, and the threats facing Standard Arabic from both the Spoken varieties and the social networking platforms that have invigorated their use.

The open-ended items on the questionnaire were attached to the multiple-choice items, as follow-on questions, to collect in-depth information about the issues presented in the original item. This method allowed participants to state their worries, objections, and hopes concerning the complicated issue of Fus ``a/Spoken dichotomy.

The process of data collection targeted Saudi speakers from both sexes, different age groups, and different educational backgrounds. The aim was to evaluate the correlation between these factors and the participants' responses to the questionnaire. A total of 2,227 participants responded to this electronic questionnaire, and the responses were categorized based on the social factors described above (e.g., age, sex, regional and educational background). Nonetheless, the age factor became the focus of analysis in this paper because of its specific significance in the survey results. The participants were categorized into six age groups: (15-20), (21-30), (31$40),(41-50),(50-60)$, and $(60+)$. The aim of this detailed subdivision is to capture all the potentially minute differences in attitudes connected to the age factor.

Several previous studies in the field of linguistic ideology have utilized questionnaires to identify and gauge participants' attitudes. These include Bouhmama and Bouhmama (2015), Shiri (2013), and Herbolich (1979). Thus, this study has built on the experiences described in these studies to formulate the questionnaire items adopted in the process of data collection.

The questionnaire used in this study was sent to five researchers in the fields of Language and Linguistics. Those researchers have provided useful comments on the formulation of some items and have confirmed the clarity and suitability of the questionnaire for its intended purpose. Additionally, the questionnaire was first disseminated among (approximately) half of the research participants (1,000 participants), and the results were calculated at that point. The second half of the participants were then allowed to respond to the questionnaire, and the results for all the participants were calculated. There was no significant difference between the two sets of results. These measures were taken to ensure the consistency and accuracy of the data collection process.

\section{Results and Analysis}

A quick look at the survey results shows two general findings regarding the society's attitudes towards using Spoken varieties. First, the survey participants clearly opposed the use of Spoken varieties in domains that have historically been associated with Fus `ha Arabic, such as delivering news and disseminating knowledge. This is evident in their rejection of news outlets that use Spoken Arabic as a medium for their content, regardless of the field or genre this content is covering (e.g., politics, sports, entertainment). The participants also opposed the use of Spoken Arabic on websites mainly used as information resources on the web, such as Wikipedia and internet forums.

The second general finding of the survey was the hesitation that surrounds the participants' responses to the items that focus on the existence of Spoken Arabic and its position as a threat to Standard Arabic. This hesitation is evident in the participants' responses to the open-ended and the multiple-choice questions on the survey. The discrepancy in responding to these items can be attributed to the influences of the ongoing campaigns against Spoken varieties, which usually call for their complete abandonment.

The survey participants, for example, strongly supported the idea that the existence of Spoken varieties is a normal thing, and that the speakers of such varieties should not be blamed for using them, which is the opposite of what the opponents of these varieties propose. However, the participants were split, almost in half, on issues such as supporting research projects on Spoken varieties and whether these varieties pose a threat to Fus $\hbar a$ Arabic. Some of the comments that were added to the responses to these items reveal the linguistic ideologies of participants who decided to share them. Thus, there are participants, for example, who state that money is better spent on research projects that study Fus ' ha Arabic rather than spending it on researching nonstandard varieties.

A comprehensive analysis of the results of this survey will focus on three main aspects of the topic: the existence of Spoken varieties within Saudi society, the domains in which these varieties should (or should not) be used, and the threats facing Fus' ha Arabic, specifically the possibility of labeling Spoken Arabic as one of these threats.

\subsection{The Existence of Spoken Arabic}

The participants were asked the following question: "Is it normal to have Spoken varieties, or is it a mistake for which speakers should be blamed?" The majority of responses $(80.7 \%)$ strongly supported the idea that it is 
normal to have a spoken nonstandard variety. This support was expressed regardless of age, sex, region, or educational background. However, the comments section that follows this item shows that some participants only gave support on the condition that the existence of a nonstandard variety should not undermine Fus ' ha, and that this variety should be used in specific domains. The fact that people can think of Spoken Arabic as normal is extremely significant; as it demonstrates awareness of the functions this variety fulfills.

The same hesitation in responding to the item regarding research funding for studying Spoken Arabic could be seen again when participants responded to this question: "Do you wish that Spoken Arabic would disappear in the future, and be replaced by Fus `ha in all aspects of life?" For this item, 54.6\% supported the switch to Fus 'ha, whereas $45.4 \%$ expressed a wish for their varieties to be maintained. However, two indicators tell us more about the nature of these responses: the first is the type of comments that participants provided, and the second is the influence of the age factor. As for the former, several participants commented that they only wish that Spoken varieties would disappear from certain fields, such as the media, education, and websites that include information (knowledge) and studies. Others specified that they do not like to see these varieties being used for writing or in official business. The participants who supported the maintenance of Spoken varieties stated that they do not wish to see them disappear because they are connected to local cultural heritage, to the identity of their tribes and hometowns, and to the memory of their "forefathers" and "their beautiful past."

With regard to age, in the (15-20) and (21-30) age groups, the participants who expressed support for the maintenance of Spoken Arabic were in the majority. The greatest difference was in the (21-30) age group, with $60 \%$ of the participants against the disappearances of Spoken varieties. In the older age groups, ranging from (31 to $60+)$, the situation was the opposite. In these groups, the majority of participants supported a complete switch from Spoken to Fus 'ћa Arabic. The greatest difference between the opposing views in this item can be seen in the (41-50) age group, with $63 \%$ of the participants supporting a complete abandonment of Spoken varieties. The overarching trend in the responses to this item indicates a greater acceptance of Spoken Arabic by younger participants, and a tendency among members of older age groups to maintain positions that view Spoken varieties negatively.

This same trend can be seen in the responses to the item regarding research funding for studies that examine Spoken Arabic. Younger participants, in general, expressed more support for this type of research, compared with older participants. In the (15-20), (21-30), and (31-40) age groups, the majority of participants said they would support this kind of research funding, with the greatest margin in the (21-30) age group, in which $58 \%$ of the members supported this view. The difference between this item and the item discussing the abandonment of Spoken Arabic lies in the fact that the (31-40) age group joined other groups with majority support for research funding. However, the comments section provides more insight into the reasons behind such responses. Several members of this group commented that any research on Spoken varieties should not undermine Fus " ha Arabic, and that it should only be for such purposes as "comparison," "criticism," "understanding local societies," and "outlining the similarities and differences between varieties."

It can be said that there is a general attitude towards Spoken Arabic (one that compares it with Fus `ha) and a specific attitude that focuses on domains of use and contexts. Although the former is usually negative within Saudi society, the latter is open for debate and discussion and, in many cases, can be positive. However, not all Arabic societies have the same general negative attitudes towards local Spoken varieties. For example, in Egypt, it is common to come across openly expressed positive attitudes towards Spoken Egyptian Arabic, including views that identify it as being beautiful, as has been discussed earlier in Haeri (2003, p. 37).

\subsection{Domains for Using Spoken Arabic}

Although the use of Spoken Arabic in the printed press is establishing some presence in certain Arab societies, this issue is considered completely unacceptable within Saudi society, on both the official and the public levels. The same is true for using Spoken varieties in broadcasting news-based programing. The idea of adopting Spoken Arabic when showing news bulletins and talk shows that focus on political issues is becoming more widespread in countries such as Egypt and Lebanon. The shows that use local varieties for discussing current affairs and light news in the Saudi context are more common now, but this does not extend to serious news programs. This type of programing is usually delivered exclusively in Fus `ha Arabic, with occasional minor code switching in limited circumstances, such as saluting the weather presenter before handing the spotlight over to him/her, or adding a light-hearted comment at the end of a report.

Abdalla (2010) presented several examples of the use of Spoken Arabic in official media outlets, both on TV and in print, in countries such as Egypt, Tunisia, and Lebanon. In the Egyptian context, we see examples from newspapers such as Al-Dustur, Al-Jumhuriyya, and Al-Ahram. In Lebanon, there is the famous example in which 
Spoken Lebanese Arabic is used to deliver the main news bulletin on LBC TV, and, in the Tunisian context, the examples come from Haqaiq Magazine. The use of Spoken Arabic in the formal press in Egypt and in Lebanon is mostly driven by the ongoing debate over identity within these countries. Specifically, proponents of these uses argue that their societies do not exclusively have an Arabic identity. Many Lebanese, for example, identify themselves as Phoenicians, and some Egyptians identify themselves as Coptic (including Egyptian Muslims who oppose the Arab identity).

In the questionnaire, the items discussing the domains for using the Spoken Arabic include the following questions: "Do you support the use of Spoken varieties when writing about sports, entertainment, and social news in the press?" and "Would you accept having newspapers and magazines that are printed in Spoken Arabic in any field?" The majority of participants $(80.6 \%$ and $81.9 \%$, respectively) rejected these propositions, without any exceptions based on age, sex, regional background, or educational level. In the comments section of these items, some participants explained the reasons behind their choices. One participant raised the issue of the kind of variety that could be used: "Is it going to be Qassimy Arabic (from Qassim region), Asiri Arabic (from Asir), or Janoubi Arabic (from the southern region of Saudi Arabia)?" The press in Saudi Arabia is predominantly national in its circulation. Thus, even though there are newspapers that have headquarters located in different regions of the Kingdom, the circulation and readership are always national. This means that these newspapers are not targeting a specific region. Therefore, the idea that readers in the Eastern region, for example, would be reading journalistic material written in Hijazi Arabic might not be widely acceptable among the general public.

Other comments focused on the orthographic characteristics of Spoken Arabic, stating that "only Fus 'ha can be used in writing," and that Spoken Arabic would not function properly in that capacity. However, the reality on the ground shows that Spoken Arabic is actually being used in writing, as can be seen on social networking apps, websites, and internet forums. The effectiveness of using these varieties, however, is still a matter for debate, because this practice is sometimes met with rejection, ridicule, and, in some cases, requests for clarification. Additionally, the participants who responded to the second item in this cluster suggested some exceptions to the exclusive use of Fus `ha Arabic in newspapers. These exceptions included writing about traditional storytelling, proverbs, and poetry; and discussing camel keeping, falconry, and other heritage-related issues.

The second domain in which participants were asked to consider using Spoken Arabic was that of internet forums. Saudis use this type of medium for several purposes, such as exchanging information and helping each other in matters such as IT, real estate, and automotive sales and repairs. Famous forums like Calam hawa? (Eve's World), the Digital Gate, haraj (auction), and Hawamer (a real estate and stocks forum) are all established locally, and almost exclusively serve local Saudi members. Writing posts on these forums is usually done in both Fus ' $\hbar a$ and Spoken Arabic. Thus, it was interesting to explore speakers' attitudes towards the use of language on these forums.

In the questionnaire, participants were asked the following question: "Is it acceptable to use Spoken Arabic when posting on internet forums?" Overall, $65.8 \%$ of participants were of the opinion that it would not be acceptable to do so, and there were no significant differences in the responses to this item correlated to social/demographic variables. This result may actually be a good representation of the situation on the ground. The use of Spoken Arabic on internet forums is highly condoned and tolerated because of the way this medium resembles face-to-face conversations. Thus, these forums differ from the printed press in that participants have strongly rejected the use of Spoken Arabic in the latter, which is viewed as a serious and formal medium that cannot be presented in any variety other than Standard Arabic. The subject matter of these forums may, however, be very conducive to the use of nonstandard forms, especially when the discussions are about issues speakers face in their daily lives.

The similarities between face-to-face conversations and informal online activities were explored extensively in Crystal's (2011) book, Internet Linguistics. On this topic, Crystal indicated that "email, chat, instant messaging and texting, though expressed through the medium of writing, display several of the core properties of speech" (p. 20). Crystal also talked about the similarities between informal speech and blogs, including the fact that they both share "the same fluidity of expression." Additionally, online mediums differ from formal newspapers in that they are delivered without "editorial interference," which results in "syntactic patterns that are never seen in traditional written varieties" (Crystal, 2011, p. 68). The similarities between these informal online activities and internet forums allow for the same generalization to apply when we talk about the style of the varieties used by the members of these forums.

The comments section for this item on the questionnaire may explain the drop in the level of rejection of use of Spoken Arabic from $81.9 \%$ (for the printed press) to $65.8 \%$ (for online forums). Several participants have 
expressed a certain level of openness to the use of local varieties, depending on the field and the type of user. Many participants indicated that, if these forums were academic or scientific, then the use of nonstandard Arabic would be offensive. They have also stated that such uses are acceptable if the writers are discussing informal issues. Other participants stated that some writers might use Spoken Arabic to gain more access to the audience and to expand their readership. The topics participants suggested as suitable for using Spoken Arabic included documenting oral heritage and narrating folkloric stories. However, in real life, all the day-to-day topics associated with forums such as the ones mentioned above (automotive, stock, and real estate market as well as auctioning) are actually discussed in Spoken Arabic.

The next item on the questionnaire asks about a well-known information resource for the general public: Wikipedia. Although this website has a reputation for not being the most accurate source of information (because of its editing policies), it is still popular as a free, easily accessible first stop for learning about a topic. One of the important developments regarding this website within the Arab World is the introduction of an Egyptian version that adopts Spoken Egyptian Arabic as the medium of authorship. Even though this website might be one of the first official websites to offer serious articles in Spoken Arabic, its introduction met a great deal of controversy and rejection. The fact that it blatantly encroaches on one of the domains exclusively reserved for Standard Arabic (namely serious and scientific discussions) was the main trigger for the rejection of this website in Arab societies. For example, AlJundi (2011) argued that the effort to create an Egyptian Wikipedia is destined to fail - in the same way that several previous efforts to promote written Spoken varieties have failed in the past. He further stated that the failure to produce one newspaper published in Spoken Arabic throughout history in the entire Arab World is a testament to the impossibility of such an effort. It is actually difficult to find strong voices supporting this project, and the opposition is widely expressed online, e.g., on Twitter, with hashtags like "together against Egyptian Wikipedia" (in Arabic).

Responding to the questionnaire, $69 \%$ of participants rejected the idea of creating a Saudi Arabic version of Wikipedia. The level of rejection was not influenced by any social factor (e.g., age, sex, education, or regional background). In this context, the participants again displayed a clear understanding of the different functionalities for the different linguistic varieties. In the comments section of this item, several participants indicated that they opposed this suggestion because they believe that serious and scientific websites should always be presented in Fus 'ha Arabic. For example, one participant stated that "encyclopedic websites should not be in any variety other than the Standard." Several participants expressed the same opinion on this item and indicated that "research and information websites should use a neutral variety that does not specify a certain region or background." Some participants argued that using regional varieties would cause division within society and would be a reason to ridicule these varieties. In fact, similar cases have been witnessed in the past, in which online forum members have confronted those who used Spoken Arabic with sarcasm and mockery.

Another controversial domain for the use of Spoken Arabic in Saudi society is commercial advertising. In this context, the varieties used to deliver the content of these advertisements have always triggered debates and discussions about their authenticity and legitimacy. These discussions usually revolve around two questions: Is it acceptable to use Spoken Arabic in commercial advertising, or is this a domain that should be reserved for Fus`ha Arabic? If it is a legitimate context for using Spoken varieties, are the actors playing these roles representing the speakers of these varieties authentically? The problem here is that any rejection of the latter might be confused with an opposition to the former. In the Gulf region, advertising agencies are notorious for producing ads that use quasi (unrealistic) local Gulf dialects, with actors from non-Gulf Arab countries.

Barhouma (2013) highlighted the criticism of the types of varieties used in these ads in his paper, "Linguistic Analysis of Advertising Discourse," which focused on the Saudi context. In this paper, Barhouma focused on what he described as the "negative effects" of these nonstandard uses of language. On the other hand, the criticism of the linguistic performance of advertisement actors can be seen in several news articles and reports that decry the failed attempts of those actors to use the local Saudi dialects (e.g., AsSharq Al-Awsat Newspaper: "Saudi Advertising: Which Dialect Prevails," March 18, 2007, and Riyadh Newspaper: "The Image of Saudis in Advertising," December 4, 2012). Thus, some members of society may reject the idea of using local varieties in advertising because of their attitudes towards the portrayal of these varieties and the way they are misrepresented and not because they have reservations about using Spoken Arabic instead of the Standard in this specific domain.

In the questionnaire, the participants were asked about using Spoken Arabic in commercial ads, especially the ones shown on Saudi-owned TV and radio channels. Although 55.1\% of the responses rejected the use of Spoken Arabic in this domain, $44.9 \%$ expressed acceptance of this practice. Even though the majority of the participants were against these uses, the lack of a strong trend on this topic could be connected to the debate over the quality 
of these ads, as described earlier. When compared to other domains such as newspapers and information websites (where the rejection levels are at $80 \%$ and $70 \%$, respectively), it is clear that the use of nonstandard expressions is far more condoned in this domain.

Additionally, the age factor was influential in this context as the type and direction of responses were different in certain age groups. Participants in the first two age groups, (15-20) and (21-30), showed stronger support for the use of Spoken varieties in commercial advertising. The strongest support was expressed by the youngest participants, with $64 \%$ acceptance of such uses (versus $36 \%$ rejection), followed by the second age group ( $21-$ 30 ), where the support was at $56 \%$ (versus $44 \%$ rejection). Even when the margin is considered slight in some of these cases, the bigger picture these results show indicates a gradual increase in the levels of rejection, correlated with the age of participants. The fact that the majority of Saudis are under the age of 30, approximately $67 \%$, (General Authority for Statistics, 2019), explains why advertising agencies are targeting the younger population in their ads, using language that is supposed to be relevant and appealing to the members of these age groups. These attempts have been successful in some cases, especially when agencies use authentic representations of dialects and witty content. However, if we can isolate the factors connected to the poor quality of linguistic production in these ads, the analysis of language attitudes in this domain will be more accurate.

The levels of acceptance of Spoken Arabic expressed in the response to the multiple-choice item above were also reflected in the comments section following it, although still not as prominently as the voices rejecting such uses. For example, several participants indicated that commercial advertising is a kind of entertainment, similar to movies and TV shows, which are already delivered in local spoken dialects. Other participants noted that the commercial nature of this domain, and the fact that "it targets people from all walks of life," mandates the use of a variety that is engaging and accessible to everyone, which is "understandable," in their opinion. The low quality in the portrayal of local dialects was also a recurrent theme in the participants' comments, with several remarks denouncing the "phony" local dialect that advertisement actors try to use and indicating that it was the reason for rejecting such a proposition. Nonetheless, the outright opposition to using Spoken varieties was still evident in the responses, with several participants reiterating their belief that any use of Spoken Arabic outside of face-to-face interaction is an attack on Fus ${ }^{\circ}$ ha Arabic and should be eradicated and condemned.

In a separate item, the participants had an opportunity to use words and expressions that they believed described the type of local dialects used in commercial advertising. Most of the comments contained critical observations and unflattering descriptions of such ads. These include terms like "sham," "farce," "terrible," "annoying," "tacky," "unbecoming," "disgusting," "low quality," "uncivil," "silly," "thoughtless," "regionalist," and "unrealistic." The participants who expressed acceptance of these ads did not actually show the same enthusiasm when commenting; most were very timid and restrained in their support. Those participants described these ads with terms such as "acceptable," "normal," "OK," "simple and easy," "marketing," "inevitable," and "draws attention."

\subsection{Threats to Fus 'ha Arabic}

The discussions of the role of Fus ${ }^{\circ}$ ha in society and the threats it faces are always focused on three reasons for concern within Saudi society. These are the growing use of Spoken Arabic, the corrupting influences of social media on Fus' ha Arabic, and the introduction of foreign terms and expressions into the language, which is mainly associated with the use of nonstandard Arabic. Thus, for the purposes of this paper, it is imperative to ascertain the local attitudes and trends regarding these perceived threats and assess their impact on the broader issue of language ideology in Saudi society.

In the questionnaire, the participants received the question, "Do you believe that Spoken varieties constitute a threat to Fus `ha Arabic?" The responses to this item manifest the internal split in society between the opposing sides of the debate. On the one hand, it can be difficult for some people to assign negative attributes to the variety they use for everyday affairs and associate with their heritage and traditions. On the other hand, large sections of society believe that such varieties are encroaching on the domains of Fus ' $\hbar a$ Arabic in a threatening manner.

With only $53.4 \%$ of participants supporting the view that Spoken varieties threaten Fus' ha Arabic, there does not seem to be a strong trend in this regard. The responses to this item do not vary based on sex, age, or regional background, indicating that the split on this issue is universal. As explained earlier, the strong campaigns against Spoken varieties have created a general sense that these varieties are corrupted versions of the Standard, and that they should be denounced and rejected. However, the fact that these varieties are strongly connected to the lives of society members may have stopped some participants from making this kind of association. Using these varieties in their prescribed domains (such as with friends and family) has always been an accepted practice, and 
those who do not feel guilty when using them for such purposes would not consider these varieties to be a threat to their most beloved Standard, Fus` ha Arabic.

The comments section following this item on the questionnaire sheds more light on the opinions of participants regarding this issue. Several participants indicated that the existence of the Quran serves as the ultimate assurance of the continuous preservation of Fus' ha Arabic. They also added that the divine promise for the protection of the Quran itself (in Chapter 15, verse no. 9) implicitly entails the preservation of the language in which it was revealed. Other participants commented on the concerns they have regarding young people's mastery of Fus' $\hbar a$ Arabic. They argued that the inability of young speakers to practice Fus' $\hbar a$ Arabic on a regular basis would weaken their grasp on this variety and would lead them to adopt nonstandard alternatives, even for the functions that require the use of Standard Arabic. The fear that this weakness might affect their understanding of religious texts was also cited by some participants. In contrast, a number of participants expressed satisfaction with the status of Fus `ha Arabic, indicating that, as long as it is used in government communications, education, official engagement, and on serious media outlets, there should not be any worries about the loss of this variety. Those who commented in this way emphasized the importance of differentiating between the roles of Fus 'ha Arabic and those of Spoken varieties within society, highlighting the latter's significance as a vessel for local cultural traditions and customs.

The second issue usually cited as a reason for concern in the discussions surrounding the state of Fus ${ }^{\text {ha Arabic }}$ is the influence of social media. In this context, several media commentators, and some Arabic language researchers, such as AlMuazen (2016) and Othman and Saleh (2013), emphasized the idea that large-scale use of social media applications is exacerbating the decline of Fus 'ta Arabic. Thus, based on these views, an item on the questionnaire was formulated to explore the participants' opinions regarding the impact of social media on Fus `ha Arabic.

On this topic, the double-edged-sword nature of these applications is acknowledged by the participants and in the literature. The controversial aspect is not actually the applications themselves but the nonstandard uses of language that are common when using them. Nonetheless, these applications have given Fus' ha Arabic a new platform on which it can be practiced and used. In this item, the participants were asked the question, "Do you believe that social media apps/websites have negatively affected Fus 'ha Arabic?" In general, the majority of respondents answered "yes," with 59.4\% expressing concern over the negative influences of these applications on Standard Arabic. However, this was one of the items in which responses varied based on the age of the participant. The strength of support for this proposition increases with age, which is consistent with what can be found in other items, such as commercial advertising and research funding. In the (15-20) and (21-30) age groups, there was only a slight majority of support (53\%) for the attribution of negativity to social media. This support increases gradually to the levels of $59 \%$ and $64.5 \%$ for the (31-40) and (41-50) age groups, respectively. In the (50-60) age group, support remains high at $61.5 \%$, although one needs to keep in mind that $64 \%$ of social media users in the Arab World are under the age of 30 (Salem, 2017). Thus, the older members of society might not be as familiar with the way such applications function as younger members.

The comments section following this item tells us more about the opposing opinions on this matter. Some participants maintained the view that any tools empowering the use of Spoken Arabic should be challenged and confronted. They indicated that the continuous use of Spoken varieties undermines speakers' ability to use and understand Fus" ha, and that the "stylistic beauty" of this variety is actually being weakened by the excessive use of local dialects. Other participants believed that the inability of speakers to master Fus' ha Arabic drives them towards clinging to local varieties and actively avoiding any chance they might need to use Fus ${ }^{\circ}$ ha to avoid making mistakes. This behavior, in their view, creates a cycle in which speakers who are already weak in this variety do not have any potential for improvement, because of their lack of practice. In other comments, participants also identified certain apps and programs as major culprits in what they describe as the "marginalization" of Fus `ha. These include "WhatsApp," "Instagram," and "Sony PlayStation's chat functions." One of the significant features of these platforms is that they mirror everyday interactions (if we exclude the cases where WhatsApp and Instagram are used for official business). Thus, the use of these platforms should not, in principle, trigger this type of condemnation because such uses are considered within the domain of the (L) variety in diglossic societies. Nonetheless, the constant feeling of guilt towards Fus ' ha Arabic in society prompts these negative attitudes and validates the call for harnessing the potential of any new technology in the service of Fus `ha and only Fus `ha.

In contrast, participants who believed that social media platforms might actually have a positive impact on Fus ${ }^{`}$ ha Arabic argued that the new opportunities provided by these applications allow more users to access texts and material produced in Standard Arabic. In one comment, a participant indicated that the popularity of social 
media offers a chance to spread the use of Fus ' $\hbar$ a Arabic. This concept, however, faces the obstacle of mastering this variety and the contexts of its use. Those who have the strongest presence on social media platforms (i.e., the biggest influencers) usually avoid the use of Fus ' ha because of its difficulty or because the subject matter of their content does not require the use of the (H) variety.

The most significant point that participants raised in this regard was the importance of social media platforms for fostering the habit of reading across society. Before the prevalence of social media, there was a continuous complaint about the fact that society members did not read enough. However, this has changed with the introduction of digital texts (e.g., e-books, links to articles, and specialized blogs) and the facilitation of dissemination of these texts through social media. The easy access to digital reading material has allowed more people to get into the habit of reading and, consequently, has encouraged the use of Fus 'ha, the variety in which written texts are produced.

Another issue identified as a threat to Fus ${ }^{\circ}$ ha Arabic within Saudi society was the use of foreign terms. Borrowing is a common phenomenon in languages around the world, and one of the ways in which the lexicon of a language evolves and expands. However, in the current linguistic environment of the Arabic language, recent cultural and technical borrowings have become characteristic of Spoken varieties. Such varieties might even be accused of not being truly part of the Arabic language because they are "so accepting of foreign borrowings" (Haeri, 2000). Consequently, these new borrowings have become the target of public scorn and contempt, because they represent (in the eyes of some commentators) the corrupt elements with which Spoken varieties are riddled. Additionally, those who call for a return to the use of Fus' ha view these recent borrowings as examples of the impurities that should be eliminated from the language.

Many of the foreign terms associated with technical/digital and cultural novelties are widely used in Spoken Arabic and are yet to be replaced by Arabic equivalents, even when the Arabic alternatives are available. These include, for example, words like "group" and "location" from WhatsApp, "trend" and "retweet" from Twitter, and other general terms such as "fashionista," "YouTuber," "mall," and "U-turn." These are just a few examples; the number of similar borrowings is immense. However, the question we face again is this: why do we find a discrepancy between the announced stance on recent (some would say unnecessary) borrowings, on the one hand, and the wide acceptance and prevalence of these borrowings on the other hand? In his discussion of borrowing as a linguistic phenomenon, Hudson (1996) approached this issue from a cultural perspective:

"One reason for using a word from such a language is to pretend, just for a moment, to be a native speaker with whatever social characteristics we associate with the stereotype. Another reason, of course, is that there is simply no other available word" (p. 55).

Hudson (1996) went on to say that "in some countries, all loan words are frowned upon because of their foreign association, so steps have to be taken to invent native words with the same meaning" (p. 56).

The Arabic alternatives, however, are not always embraced or adopted by speakers in a way that would eliminate the use of the borrowed terms. Thus, it is rare to find speakers who would use, for example, majmọ:aha instead of "group," or dawaran lilxhalf instead of "U-turn," outside written texts.

In the questionnaire, the participants were asked about the practice of using foreign words when speaking Arabic. They were then given examples of the most commonly used borrowings and asked again to comment on the use of such terms. In responding to the former item, $74.9 \%$ of participants expressed rejection of such practices. However, the level of rejection dropped to $53 \%$ when the participants were asked about their use of specific borrowed terms. General linguistic attitudes and deeply held beliefs drive speakers (as a principle) to reject foreign borrowings. However, when these borrowings are manifested in reality, in the form of commonly used terms, speakers come to realize how prevalent these terms are.

In the analysis process, the age factor has emerged as an important indicator in this context. The results of the first item demonstrated an increased growth in rejection, in correlation with age, with levels ranging from $66 \%$ for the youngest age group to $85 \%$ for the oldest age group. This was expected, as the use of borrowed forms is more common among younger speakers than among older ones.

The age factor was even more significant when analyzing the results of the second item on the use of recent lexical borrowings. The participants were asked about the use of specific terms, such as "food truck," "retweet," "anfel" (unfollow), "snap," or "sanneb" (use Snap Chat), and "group" (in WhatsApp). The most interesting finding was that a majority of participants in the first three age groups, (15-20), (21-30), and (31-40), actually acknowledged the use of such terms ( $64 \%$ of the first group, $59 \%$ of the second, and $54 \%$ of the third). In contrast, the majority in the other (older) three groups rejected such uses, with the level of rejection increasing 
with age. Thus, whereas $60 \%$ of participants in the (41-50) age group expressed opposition to using these specific recent borrowings, the numbers reached $84 \%$ in the $(60+)$ age group.

The comments section following these two items gave participants an opportunity to explain the motives behind their choices. In this section, many participants denounced the excessive use of recent borrowings, labeling them as "annoying," "a mark of lost identity," "destructive to Fus "ha," and "an insult to our religion and our identity as Arabs." Other participants talked about specific instances in which they felt particularly annoyed by such uses. For example, one participant discussed a mother who uses English words when speaking to her child in public to show that he goes to an international school. In another comment, a participant blamed Spoken varieties specifically because these uses mostly occur when speaking such varieties, as opposed to Fus ' ha Arabic.

In contrast, participants who accepted the use of recent borrowings expressed favorable attitudes towards this practice. These participants indicated that the need for such terms and the lack of alternatives are the factors behind the prevalence of their use. Several participants emphasized the spontaneous nature of such uses and that borrowed words add diversity to the speaker's perspective and character.

Thus, although there is a clear absence of alternatives to many of the recent lexical borrowings, there is also an evident lack of enthusiasm for using the suggested Arabic equivalents to the terms associated with certain new technological innovations. Given this complex situation, speakers find it difficult to reconcile their aspiration for preserving their beloved Standard Arabic with their need to use a large number of borrowed lexical forms that are connected to their daily lives. This conflict between attitudes and actual needs is what causes participants to respond to these items (on borrowing) in a way that reflects this discrepancy.

\section{Conclusion}

The aim of this paper is to investigate the discrepancy between the ideological beliefs regarding language in Saudi society and the use of language on the ground. Members of Saudi local speech communities are constantly surrounded by campaigns that criticize the local varieties they use in their everyday lives and associate with significant aspects of their heritage, culture, and traditions. People who do not have formal training in linguistics are not usually aware of the concept of diglossia and, thus, think that the linguistic situation in the Arab World is unique and does not exist in any other place. These views have resulted in a contentious feeling of guilt among speakers, who believe they are corrupting Standard Arabic through the use of Spoken varieties. In many cases however, those speakers do not have a choice because Fus' ha (which is usually acquired through formal education, being the $(\mathrm{H})$ in this diglossic situation) is not readily accessible to everyone, which exacerbates the feeling of guilt in expressed opinions.

However, with all the dire warnings regarding the use of Spoken Arabic, in reality, there seems to be a remarkable flourishing of local varieties. This includes the expansion of genres and contexts that depend on Spoken Arabic as a medium. Two examples of this trend are the rapid spread of the art of "Shilat" and the growing number of "folklore TV channels" within Saudi society. Thus, the question here is, how can we explain a dichotomy that involves a continuous denunciation of Spoken varieties, on the one hand, and their celebrated status among members of the public on the other hand?

To achieve such an understanding, the analysis in this paper starts with exploring attitudes towards the existence of an (L) variety. Although there is strong support for the existence of Spoken Arabic, this support does not extend to the issue of funding research on local varieties. Saudis do not want these varieties to compete with Fus `ha in any form, including eligibility for research funding. The younger participants, however, offered more support for the future maintenance of Spoken varieties, emphasizing that they would not want to see them disappear. Those speakers were also more accepting of the concept of funding research on local varieties.

The second dimension of attitude explored in this paper focuses on the domains in which Spoken varieties should (or should not) be used. In this context, participants have shown robust condemnation of any use of such varieties in the press, in knowledge and information resources, or on internet forums. It is worth noting, however, that the last domain (internet forums) did not garner the same level of objection because it mirrors, to a large degree, face-to-face communications. As for commercial advertising, the rejection of Spoken varieties was mild, with a greater emphasis on denouncing the failed portrayal of local dialects by actors, as opposed to using these dialects in the first place.

The third and final dimension discussed in this paper is the understanding of what constitutes a threat to Fus ${ }^{\circ}$ ha Arabic within Saudi society. The general acceptance of (L) varieties, discussed earlier, is manifested in the lack of strong support for identifying these varieties as a threat to Fus' ha. At the same time, one can see stronger reservations about social media platforms, with fears that nonstandard uses might encroach on the domains of 
Fus `ha Arabic. Nonetheless, these objections are mitigated by the suggestion that such platforms might have a potentially positive impact on Fus `ha by encouraging members of the public to practice using Standard Arabic.

Another threat to Fus ${ }^{\circ}$ ha discussed in this paper is the rise in the number of recently borrowed words and phrases from foreign languages (mainly English). These forms, generally associated with technical innovations and cultural trends, are registering a noticeable presence within Saudi society. Although there was vigorous rejection of the principle of adopting foreign words (as was expected), the picture changed when speakers were given specific examples of these borrowings. Acknowledging the use of some of these forms could be a result of realizing their constant presence and the fact that they have yet to be replaced by Fus ' ha Arabic alternatives.

Age has consistently been a factor in supporting Spoken varieties and the characteristics associated with them. This is manifested in the higher levels of support expressed by younger speakers for these varieties, including extending research funding to cover projects on Spoken Arabic, adopting recent lexical borrowings, and tolerating the use of local varieties in commercial advertising. The influence of the age factor can also be seen in the young speakers' lack of enthusiasm for blaming social media platforms for the decline of Fus ``a, and in their expressions of support for the maintenance of Spoken varieties.

Finally, a number of recommendations arise in the context of this paper to emphasize the most pressing concerns associated with the topic of language ideology within Saudi society. It is hoped that this paper could be a trigger for future research that aims to present a critical analysis of the different campaigns focusing on language use. The goal should be to refute claims that are not based on sound linguistic grounding and support initiatives that can help preserve and revitalize Fus `ha Arabic without negatively affecting other varieties. It is also hoped that more work can be done to locally raise awareness of the $(\mathrm{H}) /(\mathrm{L})$ dichotomy in diglossic societies, which in turn would allow more people to understand the linguistic situation in their region and embrace the diverse nature of their language use. This will encourage speakers to express pride in their local varieties and exert more effort to maintain and support these varieties, without putting Fus`ha Arabic at a disadvantage or infringing on its domains.

Spoken Arabic is the medium for a large body of local traditional literature and a vessel for a wide range of folkloric arts and tribal ceremonial rituals throughout Saudi society. Highlighting the importance of Spoken varieties will bolster their status and contribute to the efforts aimed at changing the general negative attitudes towards them. Subsequently, this will result in diverting more funding towards linguistic research that focuses on documenting and analyzing local varieties.

\section{References}

Abdalla, M. (2010). The Place of Media in the Arabic Curriculum. In R. Bassiouney (Ed.), Arabic and the Media: Linguistic Analyses and Application. Boston: Brill. https://doi.org/10.1163/ej.9789004182585.i-303.118

Al-Shahrani, S. (2016). Shilat and Tribal Racism: Modern Educational Issues. Tarbyatona. Retrieved from http://www.tarbyatona.net/include/plugins/article/article.php?action=s\&id=310

Alahmadi, S. (2016). Insight into the attitudes of Speakers of Urban Meccan Hijazi Arabic towards their Dialect. Advances in Language and Literacy Studies, 7(2). https://doi.org/10.7575/aiac.alls.v.7n.2p.249

Aldosaree, O. (2016). Language Attitudes towards Saudi Dialects. MA thesis. California State University, USA.

AlJundi, F. (2011). Informatics in our Lives: Fruits and Pitfalls. Damascus: Dra ArRiyadah.

AlMuazen, A. (2016). The Difficulties facing Arabic Language in the Era of Social Networking. Paper presented at Teaching Arabic Language in Higher Education Institutions: Hopes and Realities Conference. Zaid University Dubai.

Alrumaih, A. (2002). Najdi Perceptions of Saudi Regional Speech. MA thesis. Michigan State University, USA.

Barhouma, E. (2013). Linguistic Analysis of Advertising Discourse. Um AlQura University Journal of Language and Literature, 11, 159-205.

Bouhamama, D., \& Bouhamama, S. (2015). Attitudes of Kuwaiti University Students towards Modern Standard Arabic (MSA). College Student Journal, 49(3), 466-472.

Crystal, D. (2011). Internet Linguistics. London: Routldge. https://doi.org/10.4324/9780203830901

Ferguson, C. (1959). Diglossia. Word, 15, 325-340. https://doi.org/10.1080/00437956.1959.11659702

General Authority for Statistics. (2019). Population by Age Groups, and Gender 2019. Retrieved from https://www.stats.gov.sa/en/43 
Haeri, N. (2000). Form and Ideology: Arabic Sociolinguistics and Beyon. Annual Review of Anthropology, 29, 61-87. https://doi.org/10.1146/annurev.anthro.29.1.61

Haeri, N. (2003). Sacred Language, Ordinary People. York: New Palgrave.

Herbolich, J. (1979). Attitudes of Egyptians towards Various Arabic Vernaculars. Lingua, 47, 301-321. https://doi.org/10.1016/0024-3841(79)90077-9

Hudson, R. (1996). Sociolinguistics. Cambridge: Cambridge University Press. https://doi.org/10.1017/CBO9781139166843

Khater-Aso, S. (2012). The Dangers of Spoken Arabic, Illiteracy, and Non-Arabic Elements on Fus 'ћa Arabic in Arab Universities. Paper presented at Arabic Language and Modernity Conference, Islamic University, KSA.

Othman, N., \& Saleh, M. (2013). Problems of Arabic Language in Social Networking Sites: Applied Study on a Sample of Facebook Users. Paper presented at the Second International Conference for Arabic Language, KSA.

Salem, F. (2017). Social Media and the Internet of Things: Towards Data-Driven Policymaking in the Arab World-Potential, Limits and Concerns. The Arab Social Media Report, 7.

Schieffelin, B., \& Doucet, R. (1998). The Real Haitian Creole: Ideology, Metalinguistics and Orthographic Choices. In B. Schieffelin, K. Woolard \& P. Korskrity (Eds.), Language Ideologies: Practice and Theory (pp. 219-259). Oxford: Oxford University Press.

Shiri, S. (2013). Learners Attitudes towards Regional Dialects and Destination Preferences in Study Abroad. Foreign Language Annual, 46(4), 565-587. https://doi.org/10.1111/flan.12058

Suleiman, Y. (2004). A War of Words: Language Conflict in the Middle East. Cambridge: Cambridge University Press. https://doi.org/10.1017/CBO9780511819926

Versteegh, K. (2001) The Arabic Language. New York: Cambridge University Press.

Wadgiri, A. (2012). Fus 'ћa and Modern Arabic Dialects: A Relationship of Connection or Disconnection. Paper presented at Arabic Language and Modernity Conference, Islamic University, KSA.

Woolard, K., \& Schieffelin, B. (1994). Language Ideology. Annual Review of Anthropology, 23, 55-82. https://doi.org/10.1146/annurev.an.23.100194.000415

\section{Appendix A}

\section{Questionnaire}

1) Is it normal to have Spoken varieties, or is it a mistake for which speakers should be blamed?
a. Normal
b. A mistake for which speakers should be blamed
Comments:

2) Do you wish that Spoken Arabic would disappear in the future and be replaced by Fus `ha in all aspects of life?
a. Yes
b. No
Comments:

3) Would you support funding research on Spoken Arabic?
a. Yes
b. No
Comments:

4) Do you support the use of Spoken varieties when writing about sports, entertainment, and social news in the press?
a. Yes
b. No, Fus `ha must be used. 


\section{Comments:}

5) Do you accept having newspapers and magazines that are printed in Spoken Arabic in any field?
a. Yes
b. No
Comments:

6) Is it acceptable to use Spoken Arabic when posting on internet forums?
a. Yes
b. No, Fus' ha must be used.
Comments:

7) Would you accept having a Saudi Arabic version of Wikipedia?
a. Yes
b. No
Comments:

8) What do you think of commercial advertisements that use Spoken Arabic (e.g., ads on MBC TV and the radio)?
a. Acceptable
b. Unacceptable
Comments:

9) How would you describe the language used in these advertisements?

Comments:

10) Do you believe that Spoken varieties constitute a threat to Fus' ha Arabic?
a. Yes
b. No
Comments:

11) Do you believe that the use of social media platforms has negatively affected Fus `ha Arabic?
a. Yes
b. No
Comments:

12) What is your opinion regarding the practice of mixing Arabic and English or using English terms when speaking Arabic?
a. Acceptable
b. Unacceptable
Comments:

13) Do you use the following recent English borrowings, "food truck" / "retweet" / "anfel" (unfollow) / "snap" or "sanneb" (use Snap Chat) / "group" (in WhatsApp)?
a. Yes
b. No (please provide your alternatives in the comments section)
Comments:

14) Age:
a. $15-20$
b. 21-30
c. $\quad 31-40$
d. 41-50 

e. $51-60$
f. $60+$

15) Gender
a. Male
b. Female

16) Regional Background
a. Najd
b. Hijaz
c. Eastern Region
d. Northern Region
e. Southern Region

17) Educational Level
a. Below Elementary Certificate Level
b. Elementary Certificate
c. Intermediate Certificate
d. High School Certificate
e. University Level Certificate
f. Higher Education

\section{Copyrights}

Copyright for this article is retained by the author, with first publication rights granted to the journal.

This is an open-access article distributed under the terms and conditions of the Creative Commons Attribution license (http://creativecommons.org/licenses/by/4.0/). 